Supporting Information: "Technoeconomic Analysis of Brackish Water Capacitive Deionization: Navigating Tradeoffs Between Performance, Lifetime, and Material Costs"

\author{
Steven Hand ${ }^{1}$, Jeremy S. Guest ${ }^{1}$, \\ and Roland D. Cusick ${ }^{1 *}$
}

${ }^{1}$ Department of Civil and Environmental Engineering

University of Illinois at Urbana-Champaign, Urbana, IL 61801-2352

Pages: 15

Figures: 8

Tables: 4 
Section 1 - Supplemental Figures and Table
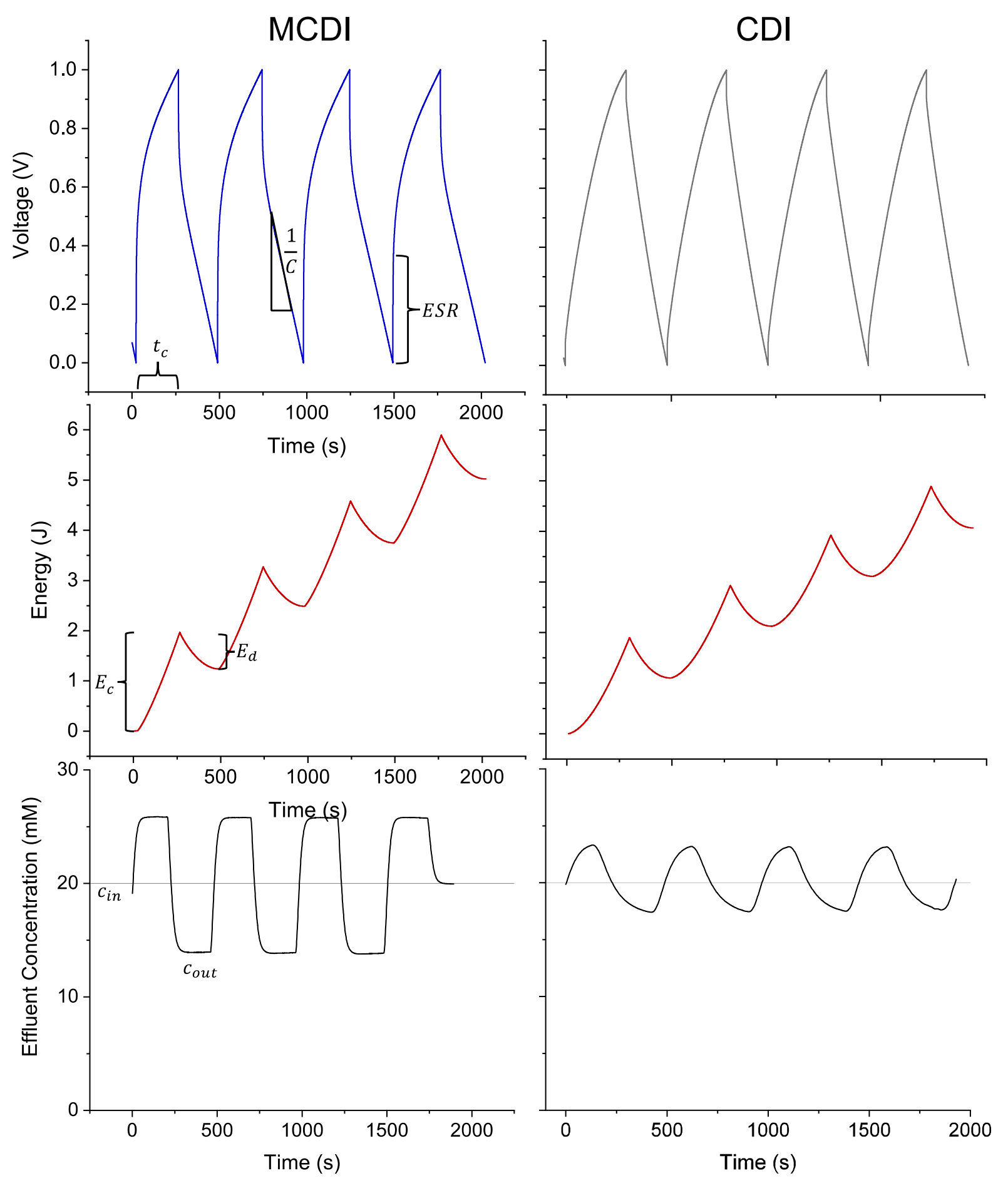

Figure S1: Representative voltage, energy, and desalination profiles from experimental MCDI and CDI cells for parameterized model validation. The time of charging, average effluent concentration during desalination, equivalent series resistance, cell capacitance, and charging/discharging energy were graphically extract for model validation. 


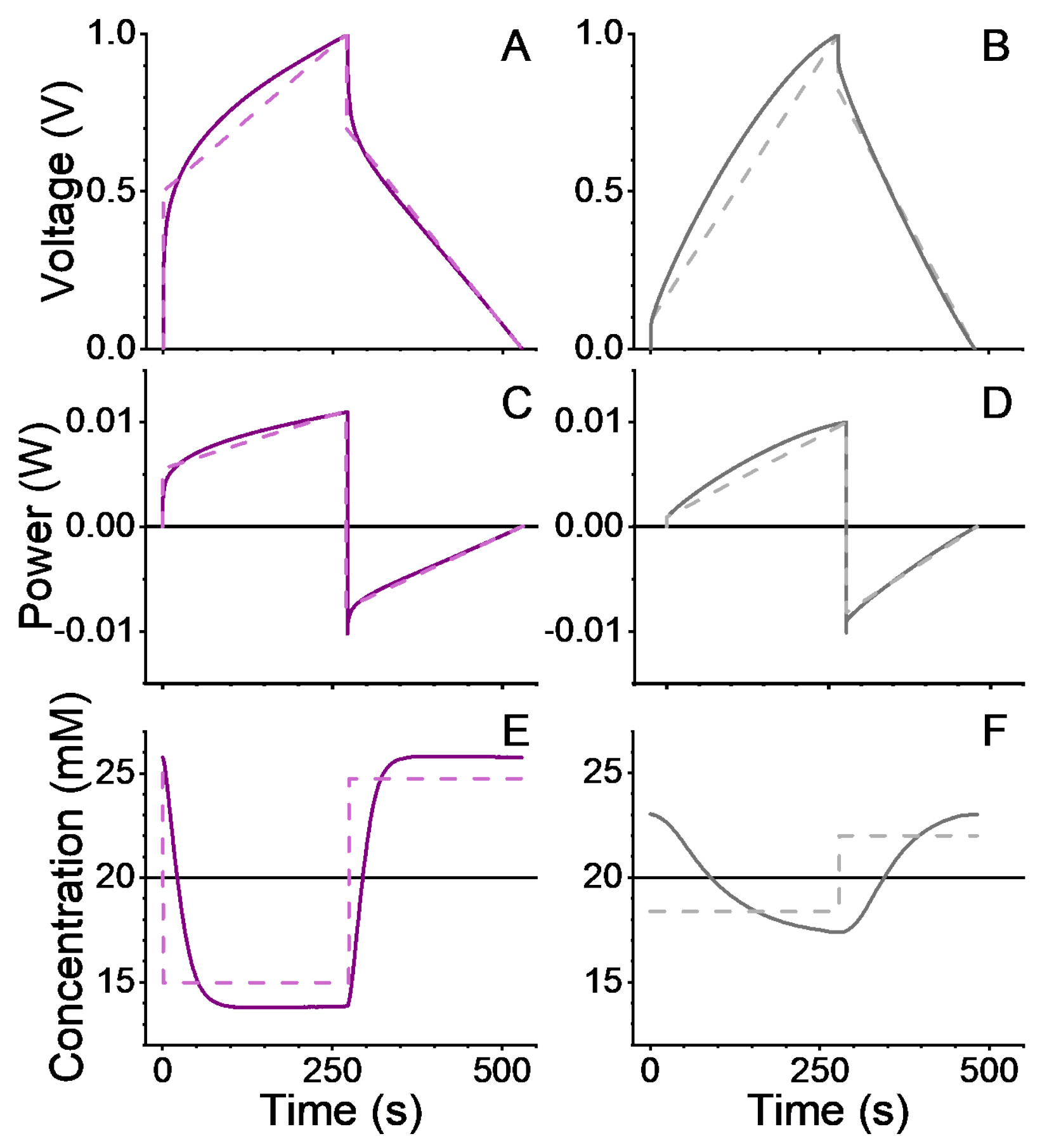

Figure S2: Calibration cell voltage, power, and concentration curves for MCDI (purple) and CDI (grey) cells for use in parameterized model validation. Experimental values shown with solid lines and corresponding model outputs shown with dashed lines. All values input to the parametrized model were drawn from individual experimental results. 

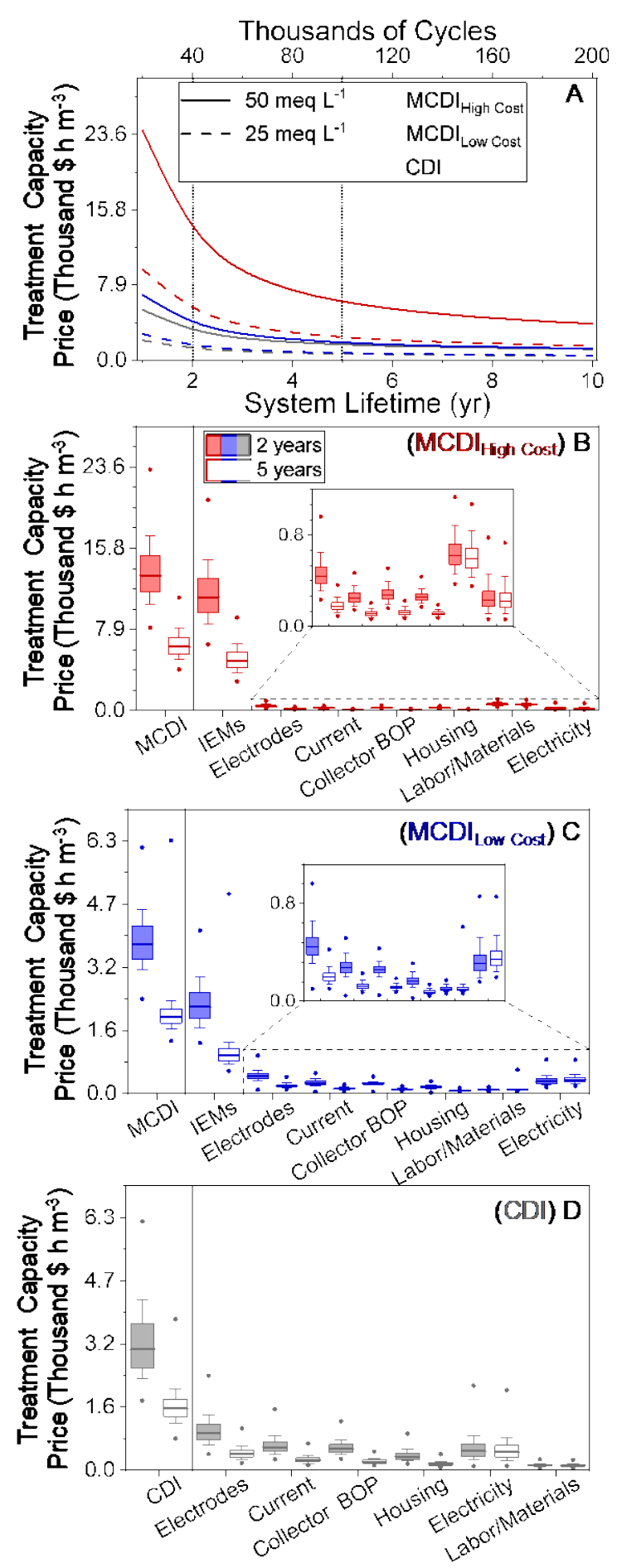

Figure S3: Median treatment capacity price ranges for CDI (grey) and MCDI with $\$ 100$ $\mathrm{m}^{-2}$ (red) and $\$ 20 \mathrm{~m}^{-2}$ (blue) IEMs at an influent concentrations of 25 and $50 \mathrm{meq} \mathrm{L}^{-1}$ as a function of system lifetime/median total cycles (A). Shaded boundaries indicate $25^{\text {th }}$ $75^{\text {th }}$ percentiles. The relative contributions of each constituent cost for CDI (D) and MCDI (B,C) at 2 (shaded) and 5 year (open) lifetimes. Boxes represent $25 \%, 50 \%$, and $75 \%$ quartiles, whiskers represent $10 \%$ and $90 \%$ quantiles, and dots represent $\min / \max$ 


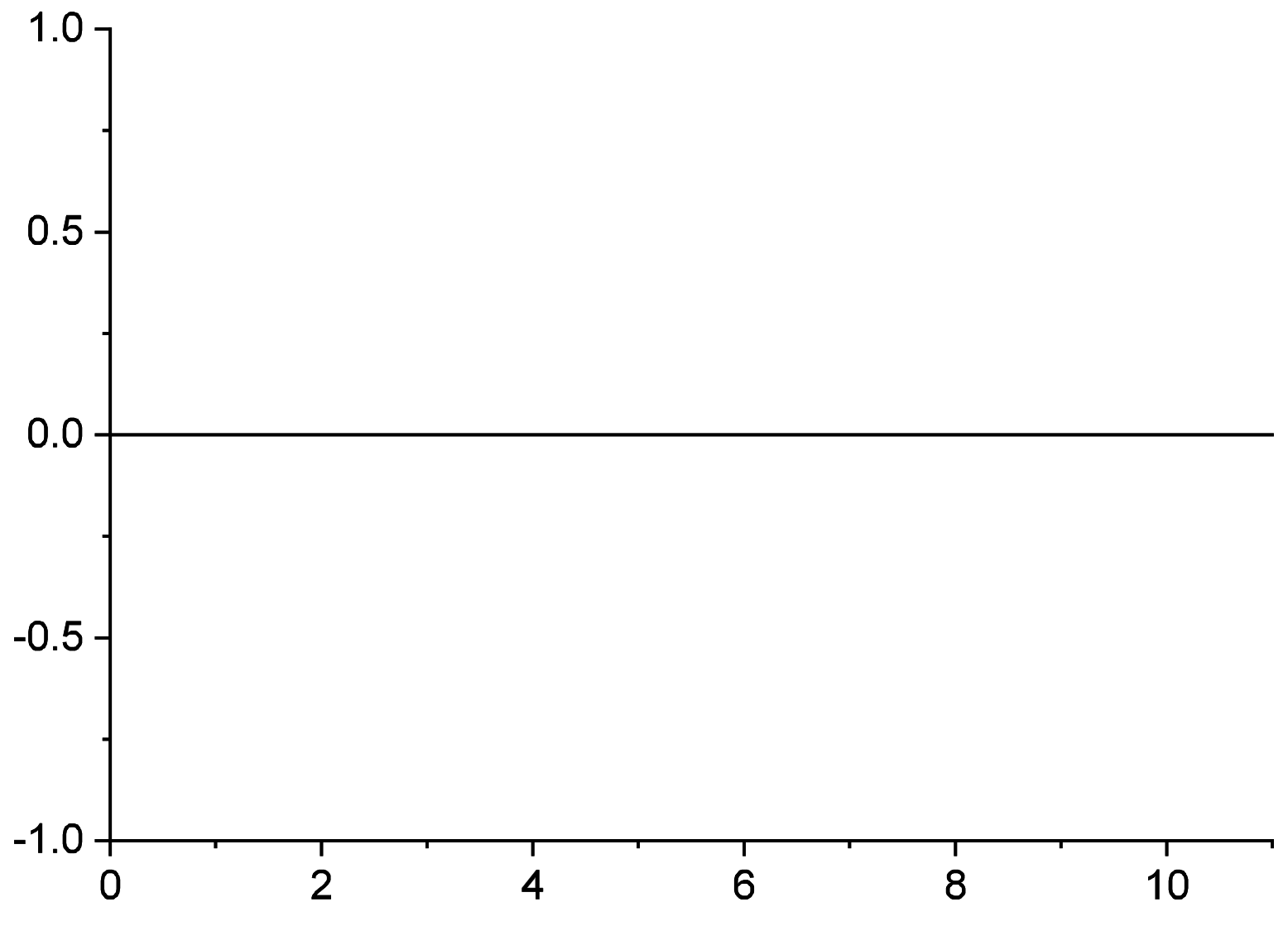

Figure S4: Mean correlation between standard performance metrics and equivalent annual water price as measured by the Spearman's rank correlation coefficient for CDI (grey) and MCDI (blue) across 25-50 mM influent concentration. Shaded region indicates standard deviation from mean across influent concentrations. 

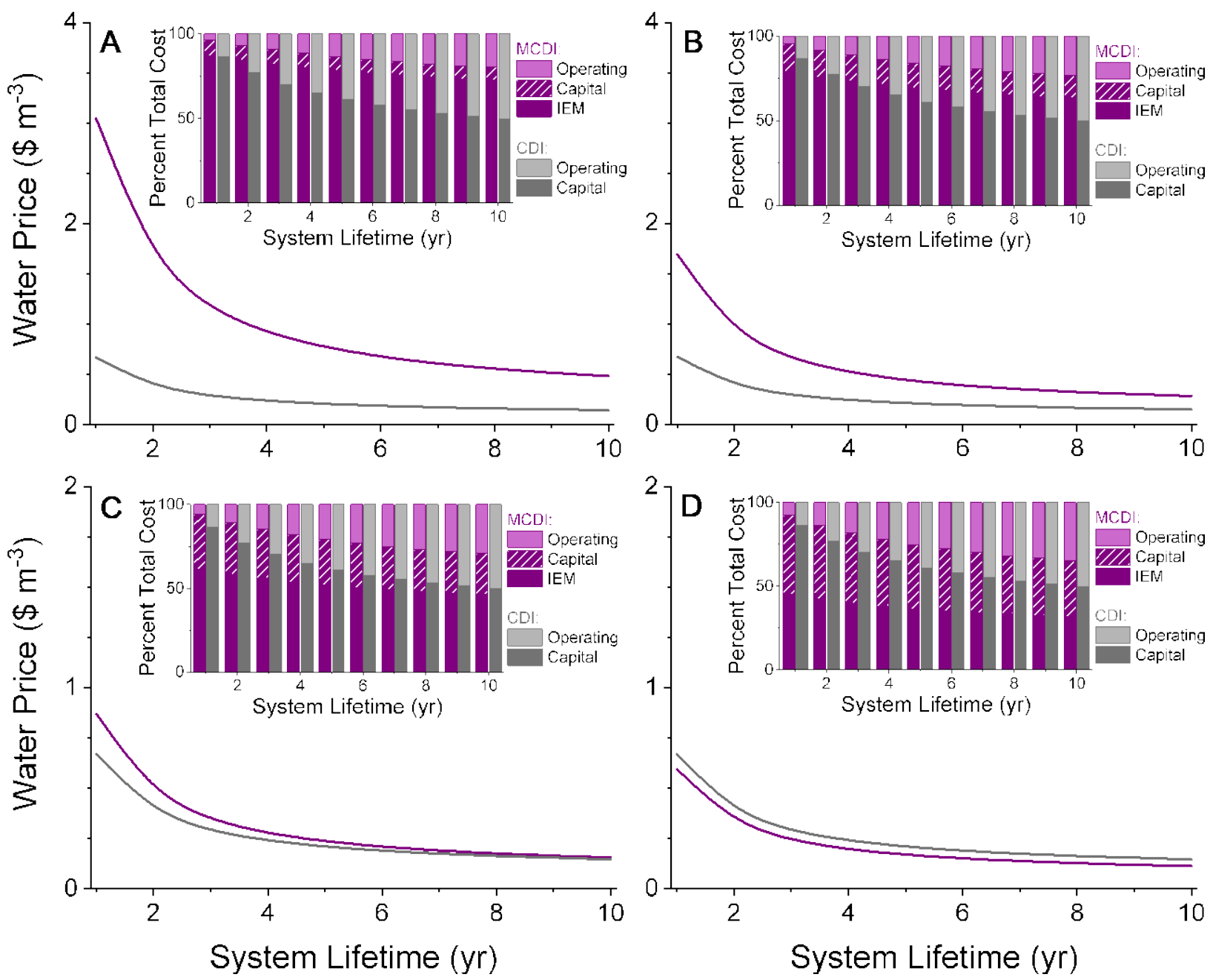

Figure S5: Median water prices for CDI (grey) and MCDI (purple) at IEM price $\$ 100 \mathrm{~m}^{-2}$ (A), $\$ 50 \mathrm{~m}^{-2}(B), \$ 20 \mathrm{~m}^{-2}$ (C), $\$ 10 \mathrm{~m}^{-2}$ (D) as sampled across the design space at varying system lifetimes. The shaded regions represent the $25^{\text {th }}-75^{\text {th }}$ percentiles. Bar charts show the relative contribution of operating and capital costs to water price (IEM costs shown separated from other MCDI capital costs). 


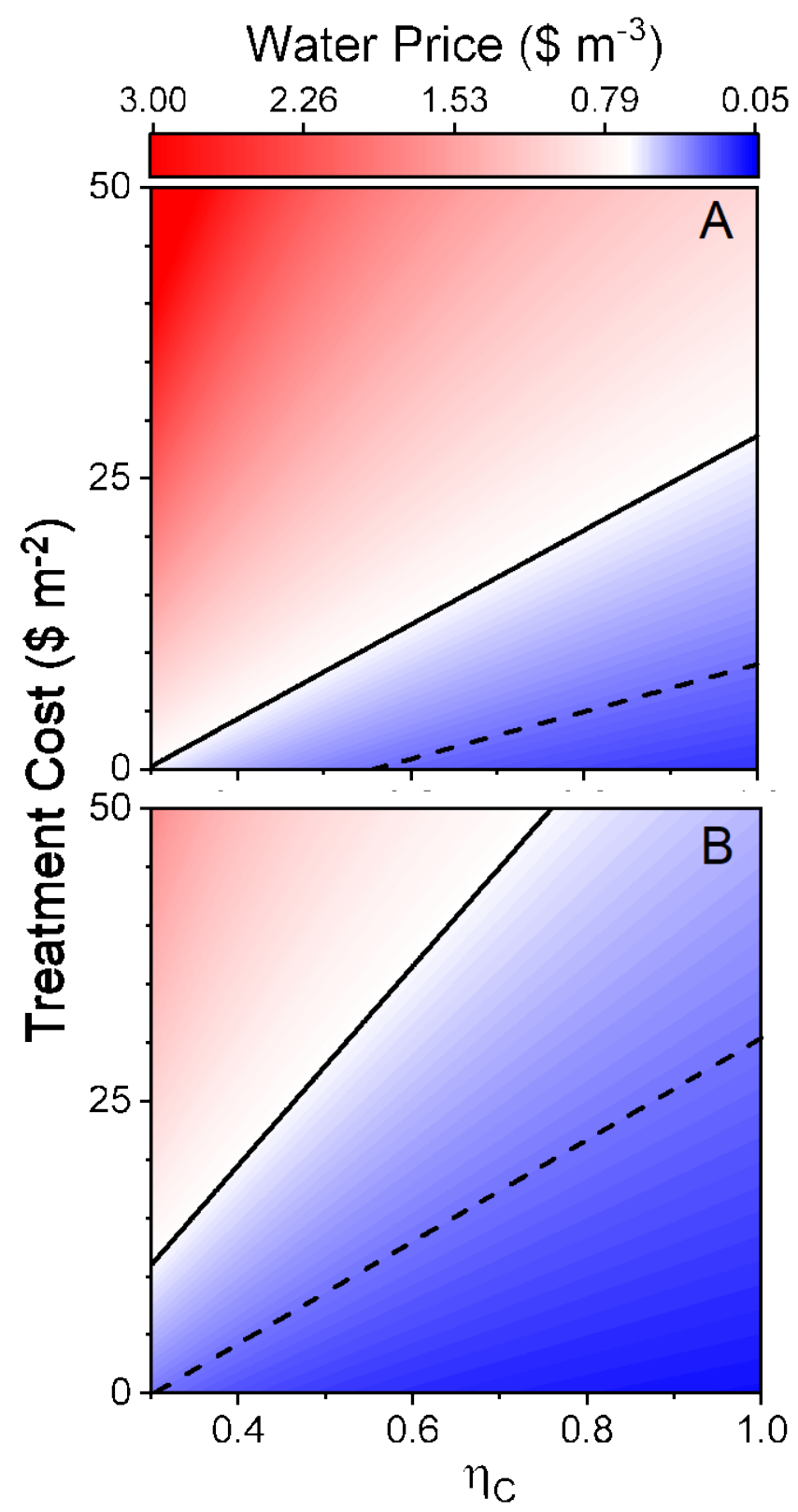

Figure S6: Impact of IEM costs on charge efficiency increases at 2 year (A) and 5 year (B) system lifetimes. Black lines display the cost of an identically sized CDI system without IEMs and 0.3 charge efficiency at 2 year (solid) and 5 year(dashed) lifetimes. The domain above a black line represents IEM prices which are not cost-effective for the given charge efficiency and lifetime. 


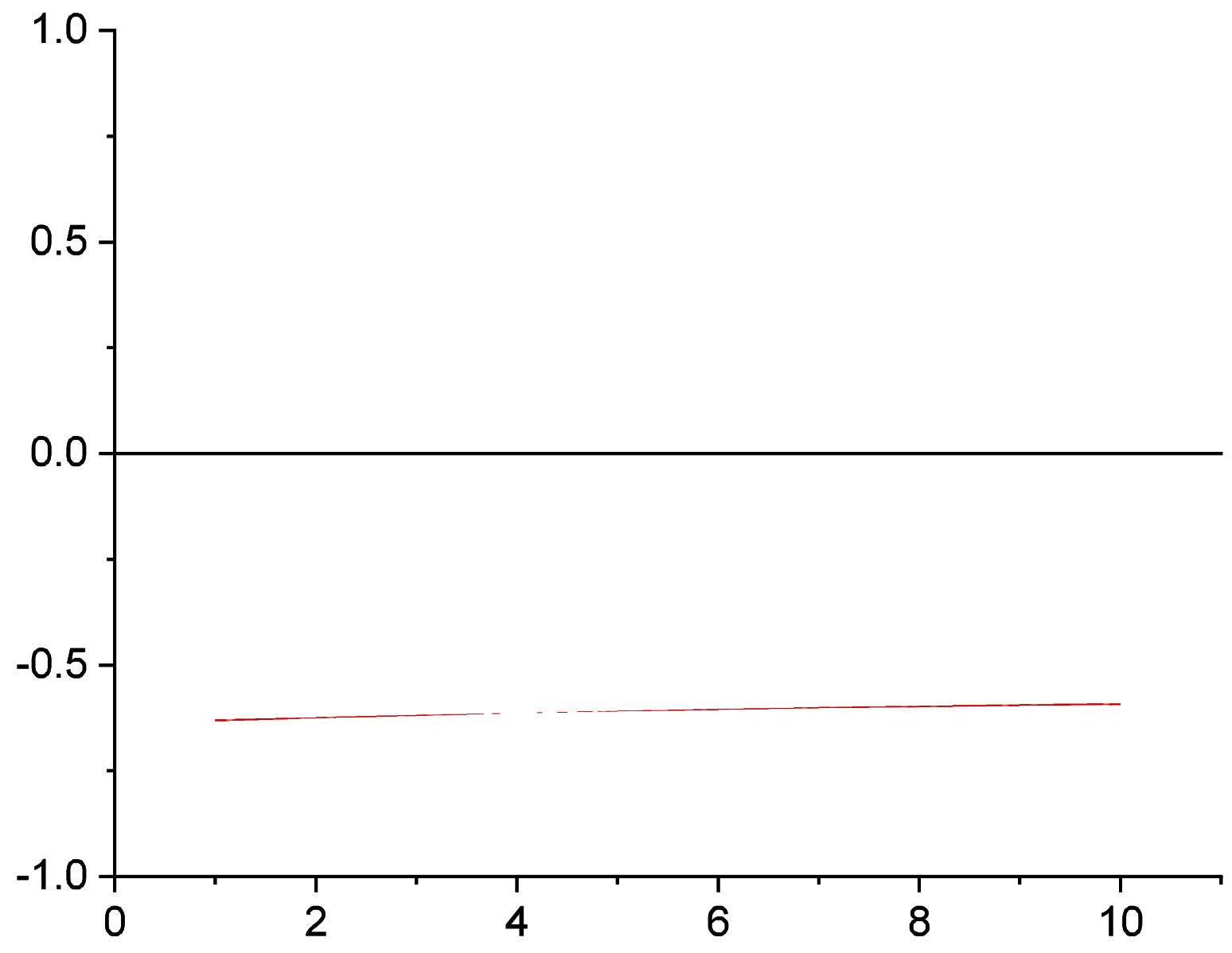

Figure S7: Mean sensitivity of unit water price to input parameters as measured by the Spearman's rank correlation coefficient for CDI (grey) and MCDI (blue) across 25-50 $\mathrm{mM}$ influent concentration. Only parameters which displayed at least moderate sensitivity $(\rho \geq 0.25)$ are shown. Shaded region indicates standard deviation from mean across influent concentrations. 

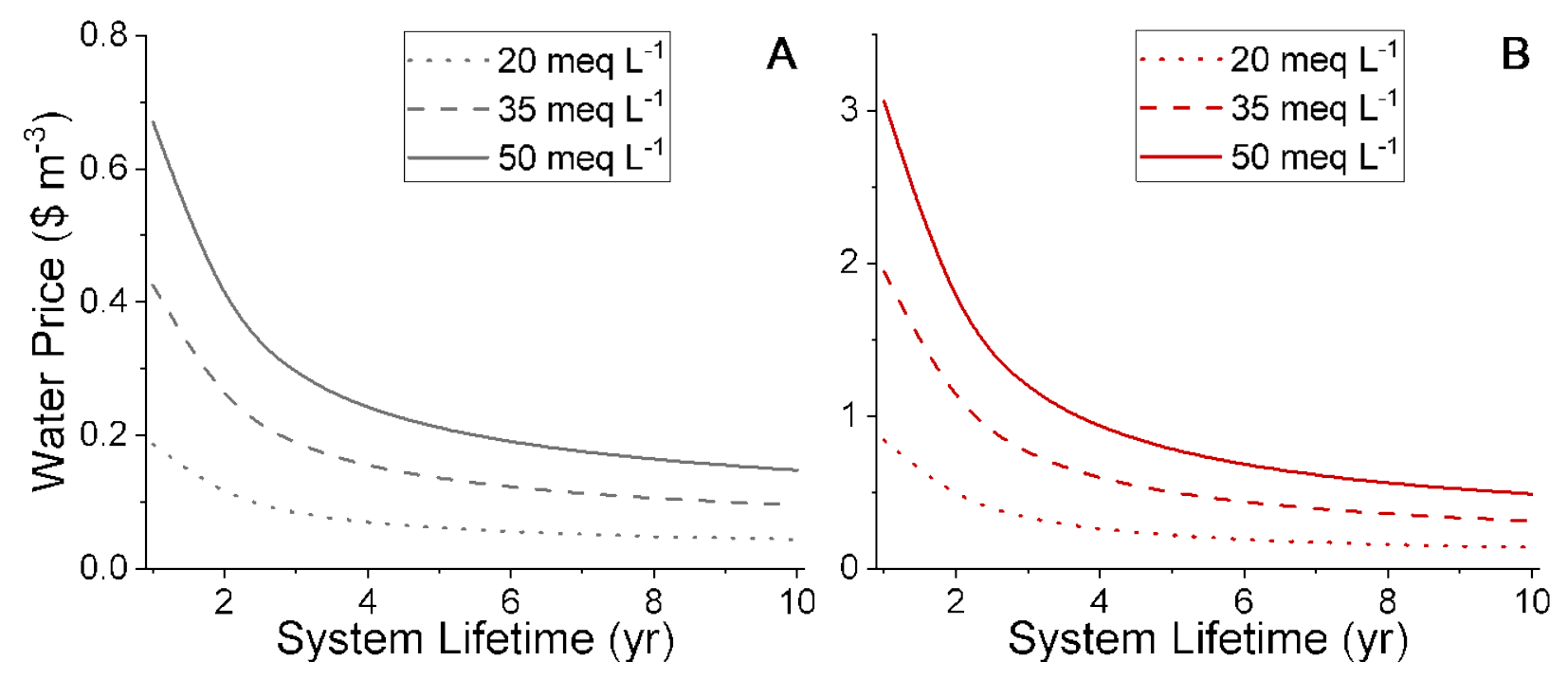

Figure S8: Median water prices for CDI (A, grey) and MCDI (B, red) 20, 35, and 50 meq $\mathrm{L}^{-1}$ influent concertation as sampled across the design space at varying system lifetimes. The shaded regions represent the $25^{\text {th }}-75^{\text {th }}$ percentiles. 
Table S1: Parameters experimentally extracted for model validation. Single cell pair $\mathrm{MCDI}$ and $\mathrm{CDI}$ systems $\left(A=20 \mathrm{~cm}^{2}\right.$ and $\left.\delta=450 \mu \mathrm{m}\right)$ were cycled with $20 \mathrm{meq} \mathrm{L}^{-1}$ influent at $50 \%$ water recovery, $5 \mathrm{~A} \mathrm{~m}^{-2}$ current density, and a $1.0 \mathrm{~V}$ cell limit until dynamic steady state was reached.

\begin{tabular}{lcc}
\hline Extracted Parameter & CDI & MCDI \\
\hline Time of Charging (s) & 280 & 270 \\
Specific Capacitance $\left(\mathrm{F} \mathrm{g}^{-1}\right)$ & 8 & 13 \\
Area-Normalized Equivalent Series & 400 & 800 \\
Resistance $\left(\Omega \mathrm{cm}^{2}\right)$ & 0.25 & 0.88 \\
Charge Efficiency & & \\
\hline
\end{tabular}


Table S2: Comparison of calculated (Cal.) vs experimental (Exp.) outputs for MCDI and $\mathrm{CDI}$ validation. Validation trials (further described in Section 2) were conducted at $50 \%$ water recovery, $1 \mathrm{~mL} \mathrm{~min}^{-1}$ flow rate, $20 \mathrm{meq} \mathrm{L}^{-1}$ influent concentration, $5 \mathrm{~A} \mathrm{~m}^{-2}$ current density, and a $1.0 \mathrm{~V}$ cell limit until dynamic steady state was reached.

\begin{tabular}{lcccc}
\hline Validation Parameter & \multicolumn{2}{c}{ MCDI } & \multicolumn{2}{c}{ CDI } \\
\hline & Cal. & Exp. & Cal. & Exp. \\
\hline Cell Pair Number & 1.1 & 1 & 1.1 & 1 \\
System Mass (g) & 378 & 400 & 435 & 406 \\
Charge Energy (J) & 1.87 & 1.96 & 1.89 & 1.98 \\
Discharge Energy (J) & 0.97 & 0.73 & 0.72 & 0.76 \\
\hline
\end{tabular}


Table S3: Inputs for parameterized model

\begin{tabular}{lccc}
\hline Input & Symbol & Value & Unit \\
\hline Total Cycle Time & $\mathrm{t}$ & $1200-1800$ & $\mathrm{~s}$ \\
Electrode Area & $\mathrm{A}$ & $70-150$ & $\mathrm{~cm}^{2}$ \\
Area-normalized equivalent & ANESR & $30-150$ & $\Omega \mathrm{cm}^{2}$ \\
series resistance & Cout & 8.5 & $\mathrm{mM}$ \\
Effluent Concentration & Cin & $20-50$ & $\mathrm{mM}$ \\
Influent Concentration & $\mathrm{Q}$ & 44 & $\mathrm{~L} \mathrm{~s}^{-1}$ \\
Flow & $\mathrm{C}$ & $20-50$ & $\mathrm{~F} \mathrm{~g}^{-1}$ \\
Specific Capacitance & $\mathrm{i}$ & $15-25$ & $\mathrm{~A}^{-2} \mathrm{~m}^{-2}$ \\
Current Density & $\eta \mathrm{CEc}$ & $0.3-0.6$ & \\
CDI Charge Efficiency & ПCEm & $0.8-1.0$ & \\
MCDI Charge Effiency & WR & $0.5-0.8$ & \\
Water Recovery & & &
\end{tabular}


Table S4: Summary statistic for simulation outputs across outputs. Ranges indicate the minimum and maximum values and parenthetical values show the mean for a given configuration. Energetic performance metrics with and without superscript $\mathrm{R}$ correspond to $0 \%$ and $100 \%$ energy recovery, respectively.

CDI

\section{$25 \mathrm{meq} \mathrm{L}^{-1} 50 \mathrm{meq} \mathrm{L}^{-1}$}

\begin{tabular}{|c|c|c|c|c|}
\hline $\begin{array}{c}\text { SAC } \\
\left(\mathrm{mg} \mathrm{g}^{-1}\right)\end{array}$ & $\begin{array}{c}4.63-21.5 \\
(11.4)\end{array}$ & $\begin{array}{c}4.73-21.5 \\
(11.5)\end{array}$ & $\begin{array}{c}12.1-37.8 \\
(23.3)\end{array}$ & $\begin{array}{c}13.0-38.4 \\
(23.9)\end{array}$ \\
\hline $\begin{array}{c}W \\
(k W)\end{array}$ & $\begin{array}{c}79.9-304 \\
(128)\end{array}$ & $\begin{array}{c}202-933 \\
(342)\end{array}$ & $\begin{array}{c}47.8-111 \\
(61)\end{array}$ & $\begin{array}{c}118-342 \\
(158)\end{array}$ \\
\hline $\begin{array}{c}E_{v} \\
\left(\mathrm{kWh} \mathrm{m}^{-3}\right)\end{array}$ & $\begin{array}{c}0.50-1.71 \\
(0.78)\end{array}$ & $\begin{array}{c}1.26-3.93 \\
(1.97)\end{array}$ & $\begin{array}{c}0.30-0.64 \\
(0.37)\end{array}$ & $\begin{array}{c}0.73-1.44 \\
(0.91)\end{array}$ \\
\hline $\begin{array}{c}E_{v}^{R} \\
\left(k^{R} ~ m^{-3}\right)\end{array}$ & $\begin{array}{c}0.11-1.26 \\
(0.46)\end{array}$ & $\begin{array}{c}0.21-3.33 \\
(1.01) \\
\end{array}$ & $\begin{array}{c}0.07-0.47 \\
(0.22)\end{array}$ & $\begin{array}{c}0.13-1.15 \\
(0.47)\end{array}$ \\
\hline $\begin{array}{c}\mathrm{P} \\
\left(\mathrm{L} \mathrm{hr^{-1 }} \mathrm{m}^{-2}\right)\end{array}$ & $\begin{array}{c}5.00-26.0 \\
(12.9)\end{array}$ & $\begin{array}{c}1.80-10.2 \\
(4.98)\end{array}$ & $\begin{array}{c}13.8-44.0 \\
(26.3)\end{array}$ & $\begin{array}{c}5.42-17.4 \\
(10.4)\end{array}$ \\
\hline$\eta$ RTE & $\begin{array}{c}0.00-0.79 \\
(0.43)\end{array}$ & $\begin{array}{c}0.00-0.85 \\
(0.50)\end{array}$ & $\begin{array}{c}0.00-0.79 \\
(0.42)\end{array}$ & $\begin{array}{c}0.00-0.84 \\
(0.49)\end{array}$ \\
\hline$\eta \top$ & $\begin{array}{c}0.01-0.05 \\
(0.03)\end{array}$ & $\begin{array}{c}0.02-0.07 \\
(0.04)\end{array}$ & $\begin{array}{c}0.04-0.09 \\
(0.06)\end{array}$ & $\begin{array}{c}0.05-0.12 \\
(0.08)\end{array}$ \\
\hline$\eta \tau^{*}$ & $\begin{array}{c}0.02-0.16 \\
(0.06)\end{array}$ & $\begin{array}{c}0.02-0.28 \\
(0.08)\end{array}$ & $\begin{array}{c}0.04-0.25 \\
(0.11)\end{array}$ & $\begin{array}{c}0.06-0.44 \\
(0.17)\end{array}$ \\
\hline
\end{tabular}




\section{Section $2-C D I$ and MCDI Experimental Validation}

The CDI cathode slurry was composed of $87.5 \mathrm{wt} . \%$ powder activated carbon (AC) (DARCO®, -100 mesh particle size), 7.5 wt.\% sodium carboxymethyl cellulose (CMC, average molecular weight $\sim 250,000$, degree of substitution 0.7 ) binder, and 5 wt.\% carbon black conductivity additive (Alfa Aesar, Tewksbury, MA) dissolved in deionized water. The anode slurry was composed of $85 \mathrm{wt} . \%$ powder activated carbon, $10 \mathrm{wt} . \%$ low molecular weight chitosan (CS, 75-85\% deacetylated) binder, and 5 wt.\% carbon black conductivity additive dissolved in a $2.5 \%$ acetic acid solution (glacial, $\geq 99.85 \%$ ).

The MCDI electrode slurry was composed of $85 \mathrm{wt} . \%$ powder activated carbon, 10 wt.\% Poly(vinylidene fluoride) (PVDF) binder, and 5 wt.\% carbon black conductivity additive dissolved in $N, N$-dimethylacetamide (DMAc) (ReagentPlus ${ }^{\circledR}, \geq 99 \%$ ). Electrodes were cast in a graphite plate current collector and dried at $353 \mathrm{~K}$. The CMC-bound cathodes were additionally cured at $403 \mathrm{~K}$ for two hours. The flow channel was cut out from a silicone gasket (SS-0.016-67909, AAA-Acme Rubber CO.). A potentiostat galvanostat (VMP3, Biologic) was used to apply and record electrical signals. For MCDI, electrodes were separated from the flow channel by cation and anion exchange membranes (CEM and AEM Type-I, Fujifilm, The Netherlands).

A syringe pump (PHD 2000, Harvard Apparatus) was used to apply a constant $1 \mathrm{~mL} \mathrm{~min}^{-1}$ flow of $20 \mathrm{meq} \mathrm{L}^{-1} \mathrm{NaCl}$ solution. The cell was cycled at $50 \%$ water recovery, $5 \mathrm{~A} \mathrm{~m}^{-2}$ current density, and a $1.0 \mathrm{~V}$ cell limit until dynamic steady state was reached (typically after 4 cycles). The conductivity in the effluent was continuously measured and recorded using a flow- through conductivity sensor that has an internal volume of $93 \mu \mathrm{L}$ (ET908, EDAQ). A standard calibration curve was used to calculate the 
salt removed per cycle. The results of experimental cycling were then compared with experimental cycling behavior using the same input parameters and experimentally recorded extracted charge efficiency and cycle length. The required number of cell pairs, total system carbon mass, and energy consumed during charging/discharging were compared for parameterized validation. 